\title{
Terapia nutricional y cirugía
}

\author{
Nutritional therapy and surgery \\ Terapia nutricional e cirurgia
}

\author{
Charles E. Bermúdez Patiño*
}

https://doi.org/10.35454/rncm.v3n1.011

La preocupación por una terapia nutricional adecuada y oportuna ha sido manifiesta y documentada desde 1500 antes de Cristo, cuando los Egipcios aportaban nutrientes y medicamentos en forma de enemas por vía rectal $^{(1)}$ con el fin de recuperar o evitar la muerte de los soldados heridos en guerra. Desde entonces es evidente el desarrollo de conocimiento, técnicas e instrumentos que permitieron el suministro de alimento al tracto digestivo y al torrente sanguíneo ${ }^{(1,2)}$.

En cuanto a los requerimientos nutricionales, el desenvolvimiento de métodos para la medición de la urea (Folin, 1905), fórmulas para estimar el gasto energético en reposo (Harris y Benedict, 1919), descripción del aumento en la pérdida de nitrógeno de acuerdo con la magnitud de la injuria (Cuthberson, 1932), el concepto de hiperalimentación en pacientes con cáncer (Tui, 1944), aportes de 30 a $46 \mathrm{Kcal} / \mathrm{Kg}$ para lograr balances de nitrógeno positivos en pacientes que requerían gastrectomía o craneotomía (Riegel, 1947) ${ }^{(1-3)}$, y muchos más fueron los pilares para la estimación de los requerimientos nutricionales como la entendemos hoy en día.

Es de suma importancia la identificación del paciente hospitalizado como una persona en condición de fragilidad, por lo que la terapia médica nutricional debe cumplir con características de: pertinencia, oportunidad,

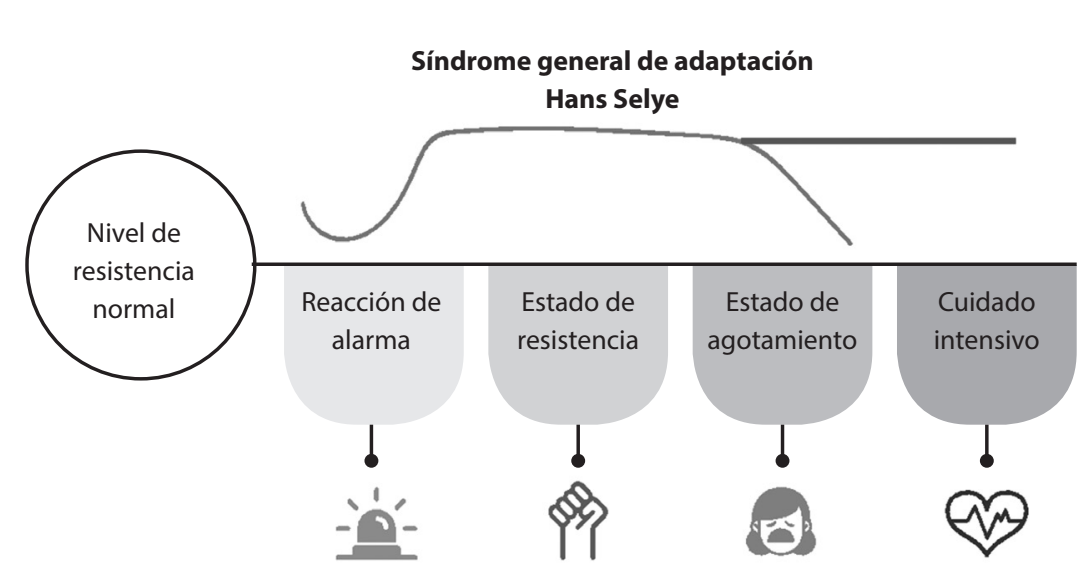

Figura 1. Síndrome General de Adaptación ${ }^{(5)}$.

suficiencia y calidad, reconociendo el derecho que le asiste, y al personal de salud como garantes de ese derecho ${ }^{(4)}$.

El doctor Hans Selye en 1936 describió el "Síndrome general de adaptación" y el origen de la palabra "stress", como un mecanismo sistémico que condicionaba una respuesta a los agentes nocivos, el cual estaba dividido en tres fases: reacción de alarma, resistencia y agotamiento (Figura 1), y la intervención médica, dentro de ella la terapia nutricional como la capacidad para prolongar el periodo de resistencia y lograr la cura ${ }^{(5)}$.

El conocimiento claro de la fisiología humana normal y las desviaciones que la respuesta metabólica al estrés y el ayuno generan, son requisitos indispensables para establecer una adecuada terapia médica nutricional, la identificación del ambiente hormonal al que se encuentra sometido el paciente, permitirá la correcta

Síndrome general de adaptación

Hans Selye

* presidencia@nutriclinicacolombia.org 
implementación de dietoterapia, suplementación nutricional oral, nutrición enteral o parenteral.

En el paciente quirúrgico, en el periodo perioperatorio, una adecuada terapia nutricional es de vital importancia, reconociendo al paciente en estado de malnutrición antes de la cirugía, iniciando una adecuada terapia médica nutricional con los sustratos necesarios que han mostrado beneficio, disminuyendo el ayuno previo a la cirugía, utilizando una carga de carbohidratos dos horas antes del procedimiento quirúrgico para disminuir la resistencia a la insulina y todos los efectos deletéreos que esta conlleva, reiniciando la vía oral y la terapia nutricional de manera temprana en el postope- ratorio y monitoreando los resultados de la intervención nutricional $^{(6-7)}$.

Además de proporcionar herramientas basadas en la evidencia para lograr los mejores resultados en nuestros pacientes, objetivo final de todas nuestras intervenciones, este número de la Revista de Nutrición Clínica y Metabolismo rinde un sincero homenaje a dos gigantes y pioneros de la Nutrición Parenteral: los doctores StanleyJ. Dudrick y José Félix Patiño Restrepo. Toda la admiración y reconocimiento a estos cirujanos que no solo dedicaron su vida a los pacientes que no podían alimentarse por vía intestinal, sino que dejaron un legado de perseverancia, caballerosidad, sencillez y amistad (Figura 2).
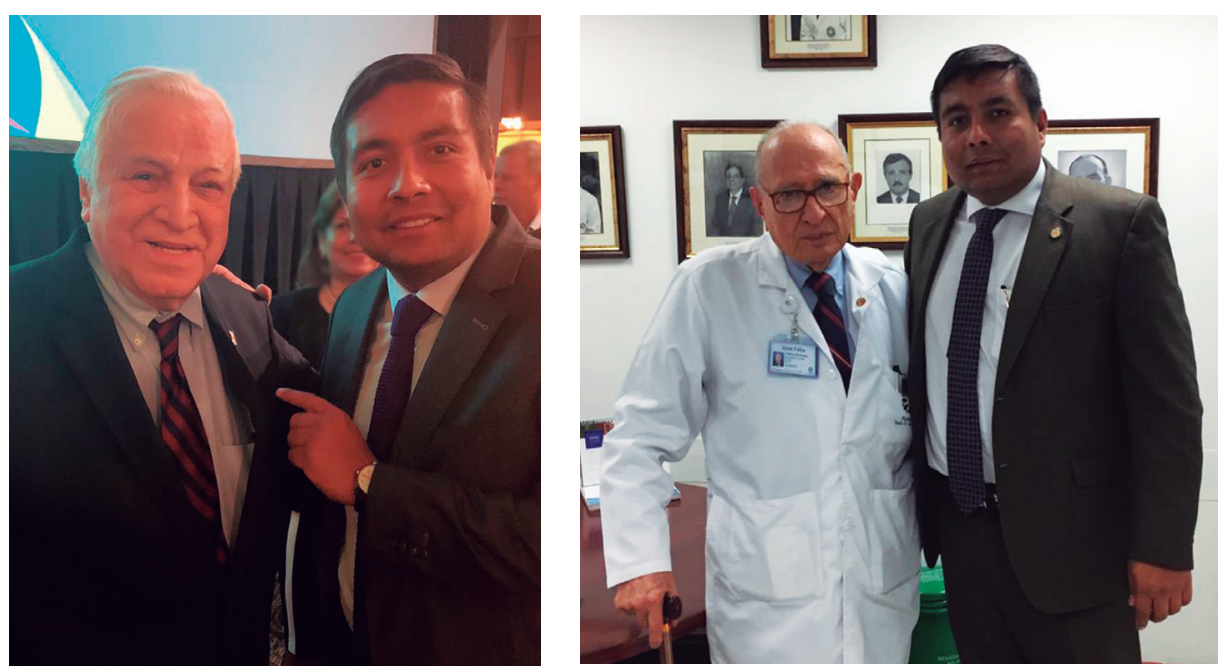

Figura 2. El doctor Charles E. Bermúdez con el doctor Stanley J. Dudrick (izquierda) y con el doctor José Félix Patiño Restrepo (derecha).

\section{Referencias bibliográficas}

1. Dudrick S, Palesty A. Historical highlights of the development of enteral nutrition. Surg Clin N Am. 2011; 91:945-64.

2. Dudrick S, Palesty A. Historical highlights of the development of total parenteral nutrition. Surg Clin N Am. 2011; 91:693-717.

3. Pellett P. Food energy requirements in humans. The American Journal of Clinical Nutrition. 1990;51(5):711-22.

4. Cárdenas D, Bermúdez $\mathrm{CH}$, Echeverri S, Pérez A, Puentes M, López M, et al. Declaración de Cartagena. Declaración Internacional sobre el Derecho al Cuidado Nutricional y la
Lucha contra la Malnutrición. Nutr Hosp. 2019;36(4):97498. http://dx.doi.org/10.20960/nh.02701.

5. Bertóla D. Hans Selye y sus ratas estresadas. Medicina Universitaria. 2010;12(47):142-3.

6. Weimann A, Braga M, Carli F, Higashiguchi T, Hübner M, et al. ESPEN guidelines: Clinical nutrition in surgery. Clinical Nutrition. 2017;36:623-50.

7. Lassen K, Coolsen M, Slim K, Carli F, Aguilar-Nascimento J, et al. Guidelines for perioperative care for pancreaticoduodenectomy: Enhanced Recovery After Surgery (ERAS) Society recommendations. Clinical Nutrition. 2012;31:817-30. 